\title{
Receptor kinase FERONIA regulates flowering time in Arabidopsis
}

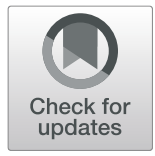

Long Wang ${ }^{1,2+}$, Tao Yang ${ }^{2 \dagger}$, Qinlu Lin ${ }^{2^{*}}$, Bingqian Wang ${ }^{1}, \mathrm{Xu} \mathrm{Li}^{3}$, Sheng Luan ${ }^{4}$ and Feng Yu ${ }^{1^{*}}$ (D)

\begin{abstract}
Background: The receptor-like kinase FEROINA (FER) plays a crucial role in controlling plant vegetative growth partially by sensing the rapid alkalinization factor (RALF) peptide. However, the role of RALF1-FER in the vegetativereproductive growth transition remains unknown. Here, we analyze the mechanism through which FER affects the flowering time in Arabidopsis.

Results: We found that the FER mRNA levels exhibit an oscillating pattern with a diurnal rhythm and that the clock oscillator CIRCADIAN CLOCK-ASSOCIATED1 (CCA1) up-regulates the expression of FER by associating with its chromatin. In addition, FER expression is regulated by clock genes, and FER also modulates the expression patterns of clock genes. Consistent with its gene expression pattern, FER positively regulates flowering by modulating the transcript accumulation and mRNA alternative splicing of certain flowering-related genes, including FLOWERING LOCUS C (FLC) and its homolog MADS AFFECTING FLOWERING (MAF). However, the RALF1 ligand negatively regulates flowering compared with FER.
\end{abstract}

Conclusions: We found that FER, which is up-regulated by CCA1, controls the flowering time by regulating the transcript accumulation and mRNA alternative splicing (AS) of some important flowering genes, and these findings link FER to the floral transition.

Keywords: FERONIA, Circadian clock, Flowering, mRNA alternative splicing, RALF1 peptide

\section{Background}

The circadian clock is a typical mechanism that is synchronized by both endogenous and external signals to regulate the vegetative-reproductive growth transition. The circadian clock consists of multiple interlocking feedback loops [1-3]. Briefly, two transcription factors, e.g., CIRCADIAN CLOCK-ASSOCIATED 1 (CCA1) and LATE ELONGATED HYPOCOTYL (LHY), are key components of the circadian clock and suppress the expression of PSEUDO-RESPONSE REGULATOR 7 (PRR7) and PRR9. In turn, PRR7 and PRR9 repress the mRNA accumulation of CCA1 and $L H Y$, resulting in the

\footnotetext{
*Correspondence: linql0403@126.com; feng_yu@hnu.edu.cn

${ }^{+}$Long Wang and Tao Yang contributed equally to this work.

${ }^{2}$ National Engineering Laboratory for Rice and By-product Deep Processing,

Central South University of Forestry and Technology, Changsha 410004,

People's Republic of China

${ }^{1}$ College of Biology, State Key Laboratory of Chemo/Biosensing and

Chemometrics, and Hunan Province Key Laboratory of Plant Functional Genomics and Developmental Regulation, Hunan University, Changsha 410082, People's Republic of China

Full list of author information is available at the end of the article
}

formation of the morning loop $[4,5]$. In the central loop, $C C A 1$ and $L H Y$ inhibit expression of the transcriptional repressor TIMING OF CAB EXPRESSION 1 (TOC1) [6], whereas the expression of TOC1 down-regulates the expression levels of $C C A 1$ and $L H Y[7,8]$. In the evening loop, TOC1 represses the expression of GIGANTEA (GI) [8], and the evening complex (EC), which includes EARLY FLOWERING 3 (ELF3), ELF4 and LUX ARRH YTHMO (LUX), suppresses the expression of TOC1, GI, and PRR9 [9, 10]. These three interlocking feedback loops are the fundamental components of the circadian clock.

Under long-day (LD) conditions, the circadian clock regulates the flowering time via the GI-CONSTANS (CO)-FLOWERING LOCUS T (FT) pathway, which is called the photoperiodic pathway [11-15]. CO is a zinc finger transcription factor that promotes flowering by directly activating $F T$ expression $[16,17]$. FT is the key regulator of the floral pathway, and diverse inputs, including $F L C$, are integrated by regulating the expression of FT [18]. FLC, a MADS-box transcription factor, has 
five homologs, MAF1 to MAF5, in Arabidopsis. FLC acts as a key repressor of flowering via the vernalization and autonomous pathways by inhibiting the expression of $F T$ and SUPPRESSOR OF CONSTANS 1 (SOC1). The mRNA expression of $F L C$ is regulated by several genes, including FCA, FY, FLK, FLD, VRN1, VRN2 and VIN3, in both the autonomous and vernalization pathways [19]. In addition, increasing lines of evidence have shown that $F L C$ precursor pre-mRNA processing plays an important role in controlling the flowering time. For example, the SC35 and SCL proteins regulate FLC splicing to control the flowering of Arabidopsis [20]. The RNA-binding proteins RZ-1B and RZ-1C have been implicated in the regulation of FLC splicing through their interaction with serine/arginine-rich (SR) proteins [21]. AtU2AF65b functions in abscisic acid-mediated flowering by regulating the alternative splicing of FLC [22]. All these data indicate that the regulation of FLC premRNA and mRNA expression is essential for flowering.

Alternative splicing (AS), which refers to the production of multiple mRNA isoforms from a single gene, regulates gene expression and increases protein diversity in eukaryotes. AS is mediated by the spliceosome, which consists of five small nuclear ribonucleoprotein particles (snRNPs) and more than 180 types of proteins [23]. Some studies have indicated that AS regulates the timing of floral transition through the integration of external environmental signals, including the ambient temperature and environmental stress. For example, cold treatment affects the abundance of two different $M A F 2$ splice variants, MAF2 var1 and MAF2 var2, which have different functions in the modulation of the flowering time [24]. In addition, FLOWERING LOCUS M (FLM) has two main splice variants, FLM- $\beta$ and FLM- $\delta$, which compete for interaction with the floral repressor SHORT VEGETATIVE PHASE (SVP). SVP interacts with the protein splice variant FLM- $\beta$ to repress flowering at low temperatures. In contrast, the DNA-binding ability of the SVP-FLM- $\delta$ complex is reduced at high temperatures to accelerate flowering [25]. Protein arginine methyltransferase 5 (PRMT5/SKB1), known as shk1 kinasebinding protein, is essential for pre-mRNA splicing. This protein dissociates from the FLC promoter, and salt stress induces increases in FLC expression, which results in late flowering [26]. External environmental signals modulate AS-induced flowering. However, the receptors that can link external environmental signals with regulation of the AS of flowering genes remains largely unclear.

The receptor-like kinase FERONIA (FER), which serves as a node for the crosstalk between plant growth and environmental cues, is a versatile regulator of plant growth and survival [27-29]. Glycosylphosphatidylinositolanchored proteins (GPI-APs), namely, LRE (LORELEI) and LLG1 (LRE-like GPI-APs), which act as chaperones/ co-receptors of FER, work together with FER after LLG1/ LER-FER perceives different RALF peptides [30-32]. The loss-of-function mutation of fer- 4 causes defects in cell elongation, leading to retarded vegetative development and shorter root hairs [33, 34], which indicates that FER promotes cell growth in some vegetative tissues. In addition, FER is involved in stress responses, such as responses to temperature, salt, and pathogens $[27,29,35-$ 37]. Therefore, FER controls cell growth and the stress response by integrating different environmental cues and endogenous factors in Arabidopsis. In this study, we found that FER, which is up-regulated by CCA1, controls the flowering time by regulating the transcript accumulation and mRNA AS of some important flowering genes and is thus linked to the floral transition.

\section{Results}

FER transcripts oscillate at a diurnal rhythm controlled by CCA1

We hypothesized that FER might be controlled by a diurnal rhythm because the transcript levels of FER gene depended on the sampling time. Thus, we analyzed the expression of FER using the web-based tool "Diurnal" (http://diurnal.mocklerlab.org/diurnal_data_finders/new) [38]. The results showed that FER exhibits a diurnal rhythm under LD, short-day (SD) and continuous-light (LL) conditions (Fig. S1A-D). According to the qPCR analysis, the expression of FER fluctuated rhythmically after transfer from 16-h light/8-h dark (LD) conditions to LL conditions and from 8-h light/16-h dark (SD) conditions to LL conditions (Fig. 1a-b). The FER oscillation lasted approximately $24 \mathrm{~h}$. After transfer from LD to LL conditions, the transcript level of FER first increased at dawn, peaked in the morning, and reached its maximum and minimum during Zeitgeber time 32 (ZT 32) and ZT 28 , respectively (Fig. 1a). In contrast, after transfer from $\mathrm{SD}$ to LL conditions, the expression of FER increased during daytime and peaked at ZT 40 (Fig. 1b). To readily assess the total FER protein (including the phosphorylated and dephosphorylated forms) levels in one band, we shortened the SDS-PAGE running time to prevent separation of the phosphorylated and dephosphorylated forms of FER [27]. According to a GFP expression analysis, the lowest and highest accumulation of the FER protein in the $p F E R$ :FER-GFP tagged plants was detected at ZT 28 and ZT 32, respectively (Fig. 1c). A similar result was obtained with the WT plants using the FER antibody (anti-FER) (Fig. 1d). The protein expression of FER was consistent with the mRNA oscillation pattern under LD conditions, and an approximately 4-h delay was observed between FER protein and mRNA expression because protein translation generally occurs after mRNA transcription. To determine whether the expression of 

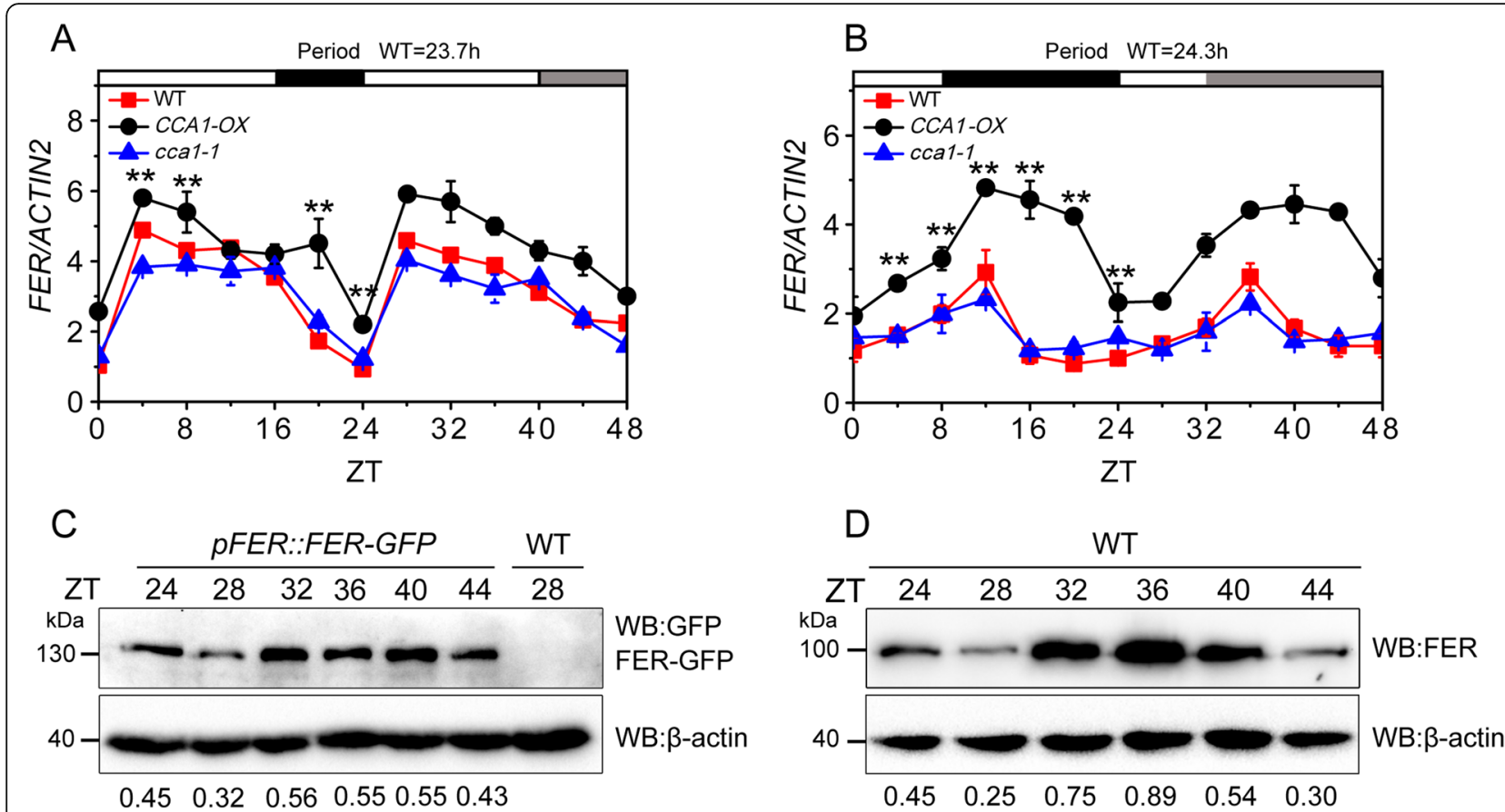

Fig. 1 FER expression oscillates at a diurnal rhythm. a FER expression in CCA1-OX, ccal-1 and WT plants under LD condition. Seedlings sampled at 4-h intervals were analyzed by QPCR. Day, night, and subjective night are denoted by white, black, and gray bars. ZT 0 represents the light-on time of the day, during which the sample was collected. ACTIN2 was used as an internal control to calculate the relative mRNA levels; the experiments were repeated three times, and the error bars represent the SD of three technical replicates. The period was analyzed using BioDare2. Asterisks indicate a significant difference between CCA1-OX and WT (**P $<0.01$, one-way ANOVA with Tukey's test). $\mathbf{b}$ FER expression in CCA1-OX, cca1-1 and WT plants under SD condition. The experiments were repeated three times, and the error bars represent the SD of three technical replicates. The period was analyzed using BioDare2. Asterisks indicate a significant difference between CCA1-OX and WT ${ }^{* *} P<0.01$, oneway ANOVA with Tukey's test). c Immunoblot analysis. The samples were collected at 4-h intervals. All proteins were extracted from pFER::FER-GFP and WT seedlings and then analyzed using anti-GFP antibodies to detect the FER-GFP protein. The FER-GFP/ $\beta$-actin ratio is displayed below the gel, and $\beta$-actin was used as the loading control. The experiments were independently repeated three times with similar results. $\mathbf{d}$ Immunoblot analysis. Protein was extracted from WT seedlings and then analyzed using anti-FER antibodies to detect the FER protein. $\beta$-actin was used as the loading control, and the FER/ $\beta$-actin ratio is displayed below the gel. At least three biological replicates were performed, and similar results were obtained.

FER is controlled by CCA1, we measured the FER mRNA levels in WT, CCA1-overexpressing (CCA1-OX) and cca1-1 mutant plants under LD and SD conditions. The FER mRNA levels oscillated in both the CCA1-overexpressing line (CCA1-OX) and the cca1-1 mutant. The expression level in the CCA1-OX plants was higher than that in the WT plants under both LD and SD conditions (Fig. 1a and b), which suggested that the expression of FER is affected by CCA1.

\section{CCA1 regulates FER expression by directly binding to its chromatin}

The mRNA expression levels of FER were significantly increased in the CCA1-OX plants under both $\mathrm{LD}$ and SD conditions (Fig. 1a and b). Because CCA1 is a transcription factor, we speculated that CCA1 might modulate FER expression by binding to the FER chromatin in Arabidopsis. Interestingly, the chromatin of FER contained an EE (AAATATCT) element (Additional file 2: Figure S2A and B), which is a candidate binding site for
CCA1 under LD conditions [39, 40]. In addition, according to the ChIP-seq data, CCA1 binds to FER chromatin $[39,40]$. To confirm that CCA1 binds to FER chromatin, we purified the GST-CCA1 protein and performed an electrophoretic mobility shift assay (EMSA). As shown in Fig. 2a, CCA1 associates with $p F E R$, which contains an EE element. We then performed a competitive EMSA with unlabeled WT and mutant (AAATATCT mutated to GGGGGGGG) probes and found that the WT probe, but not the mutant probe, competed with the labeled probe, which indicated that CCA1 specifically binds to FER chromatin. We subsequently performed chromatin immunoprecipitation (ChIP) assays using both the WT (Col-0) and 35S::CCA1-Myc line with the anti-Myc antibody. The fragments of FER chromatin containing the $\mathrm{EE}$ element were enriched in the 35S::CCA1-Myc but not the WT plants under the LD conditions, which suggested that CCA1 binds to FER chromatin in vivo (Fig. 2b and c). In addition, we performed dualluciferase (LUC) assays to determine whether CCA1 
A

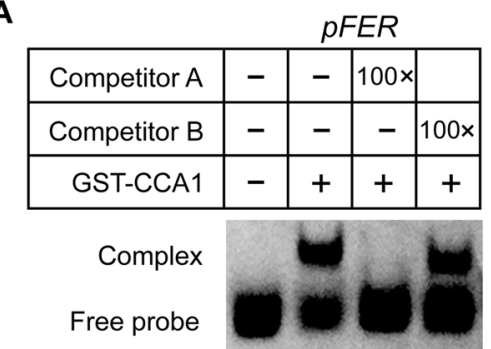

C

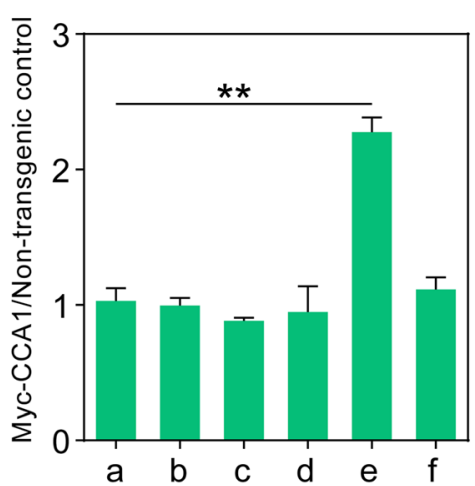

B

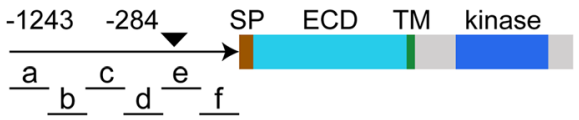

D

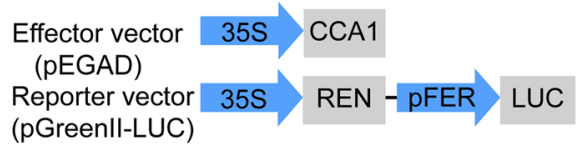

$\mathbf{E}$

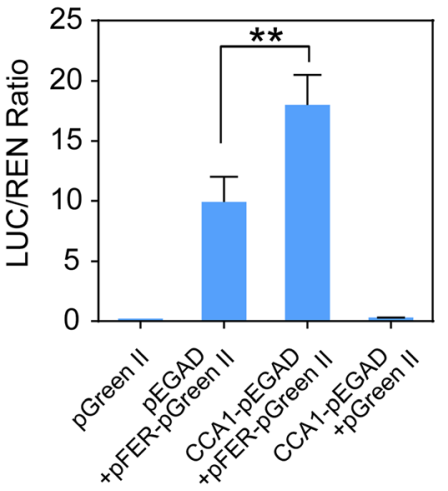

Fig. $\mathbf{2}$ CCA1 interacts with the chromatin regions of FER and promotes its expression. a EMSA was performed to confirm the binding of CCA1 to the EE motif of the FER promoter in vitro. Competitor A was the CCA1 binding motif without FITC labeling; Competitor B was the mutant EE fragment without FITC labeling. pFER: The FER fragment contained putative CCA1 binding sites. Sequences of individual DNA probes are listed in Additional file 13: Table 5. The experiments were independently repeated four times with similar results. b Diagram depicting the promoter (arrow), signal peptide (SP), extracellular domain (ECD), transmembrane domain (TM) and kinase domain (Kinase). The black triangles indicate the positions of the EE element (AAATATCT), and the black solid lines depict the DNA regions that were amplified by ChIP-qPCR. c ChIP-qPCR. ChIP assays were performed using an anti-Myc antibody. DNA regions that were amplified by qPCR are indicated by black solid lines in B. Plants were grown under LD conditions and harvested at ZT 0 . Values are relative to a non-transgenic control, and three independent experiments were performed with similar results, error bars represent the SD of three biological replicates (** $P<0.01$, Student's t-test). $\mathbf{d}$ Reporter and effector constructs used in the transient expression assays. REN luciferase was used as an internal control to normalize the values in individual assays. $\mathbf{e}$ Relative reporter activity (LUC/REN) in Arabidopsis protoplasts. Error bars represent the SD of three biological replicates, and asterisks indicate a significant difference (**P<0.01, Student's t-test).

regulates the expression of $F E R$ by directly interacting with $F E R$ chromatin. In this assay, the $C C A 1$ overexpression construct (CCA1-pEGAD) was used as the effector, and the $p F E R-L U C$ reporter ( $p F E R-p G r e e n I I)$ was the readout (Fig. 2d). As shown in Fig. 2e, the LUC activity in the CCA1-pEGAD group was higher than that in the $p E G A D$ control group, which indicated that CCA1 coexpression increases the expression of FER in Arabidopsis. Thus, CCA1 binds directly to FER chromatin and upregulates FER expression in Arabidopsis.

\section{FER mutation alters expression patterns of clock genes}

Because the expression of the FER gene exhibits a diurnal rhythm in plants, we tested whether the expression of FER is a simple output of the clock mechanism or whether $F E R$, which is a receptor kinase, also serves as a regulator of the clock mechanism that integrates extracellular signals. The expression levels of six clock genes, namely, CCA1, LHY, TOC1, PRR7, PRR9 and GI, in the WT and fer- 4 mutant were measured every $3 \mathrm{~h}$, and these genes were then analyzed using the BioDare 2 website [41]. The analysis revealed that the periods of these six genes in the fer- 4 mutant plants were shorter than those in the WT plants (Fig. 3a-f). In addition to shortening the period of the clock genes, the fer mutation altered the amplitudes of the CCA1, TOC1 and PRR7 genes under LD conditions (Additional file 3: Figure S3A-D). The amplitude of CCA1 increased by more than $70 \%$ (amplitude: $\mathrm{Col}-0=0.38$ and $f e r-4=0.68$ ) in the fer4 mutant (Additional file 3: Figure S3A). However, the TOC1 and PRR7 transcription levels decreased in the fer-4 mutant. These results indicated that FER expression is regulated by clock genes and that FER modulates the expression patterns of clock genes.

\section{FER modulates the flowering time}

CCA1 overexpression causes late flowering though the repression of GI [42], as high expression of CCA1 was observed in the fer-4 mutant (Additional file 3: Figure $\mathrm{S} 3 \mathrm{~A})$. We speculated that FER likely plays a role in the 
control of the flowering time. The flowering times of the fer-4 mutant and WT plants were examined. Under LD conditions, the fer- 4 mutant showed a significant delay in the flowering time, as measured by either the days to flowering or the numbers of leaves at flowering (Fig. 4ac). This phenotype was also observed in $\operatorname{srn}[34,43]$, which is another FER-null mutant in the ecotype C24 background (Additional file 4: Figure S4A-C). We found that fer-5, which is a knock-down mutant of FER (Duan et al., 2010), had a late-flowering phenotype (Fig. 4a-c).
To determine the molecular mechanism underlying the delayed flowering phenotype of the fer- 4 mutant, we examined the expression of GI-CO-FT, a key pathway of photoperiodic flowering. In the fer- 4 mutant, the expression of GI began to increase at ZT 4 and peaked at ZT 8 , which is similar to the findings observed in the WT plants. However, the GI peak in the fer-4 mutant was significantly lower than that in the WT plants (Fig. 4d). Similarly, the oscillation pattern of the $C O$ transcript in the WT plants was similar to that in the fer- 4 mutant,
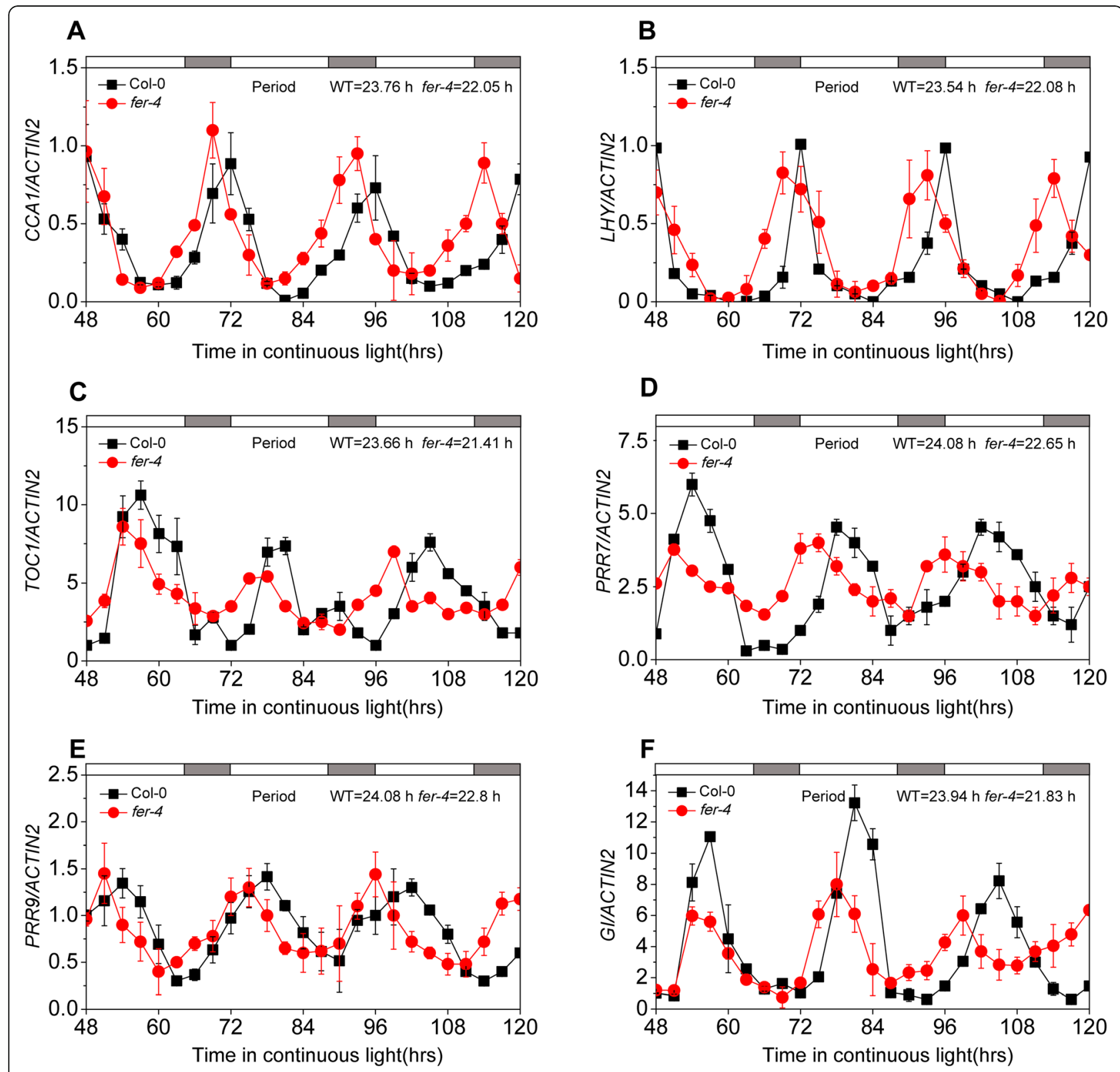

Fig. 3 FER mutation affects the expression patterns of the clock genes. The expression levels of CCA1 (a), LHY (b), TOC1 (c), PRR7 (d), PRR9 (e) and GI (f) transcripts in WT and fer-4 mutant plants under LL conditions are shown. Seven-day-old seedlings were grown under LD conditions, transferred to LL at ZT 0, sampled at 3-h intervals and analyzed using qPCR. The period was analyzed using BioDare2. All experiments were performed at least three times with similar results, and the error bars represent the SD of three technical replicates 


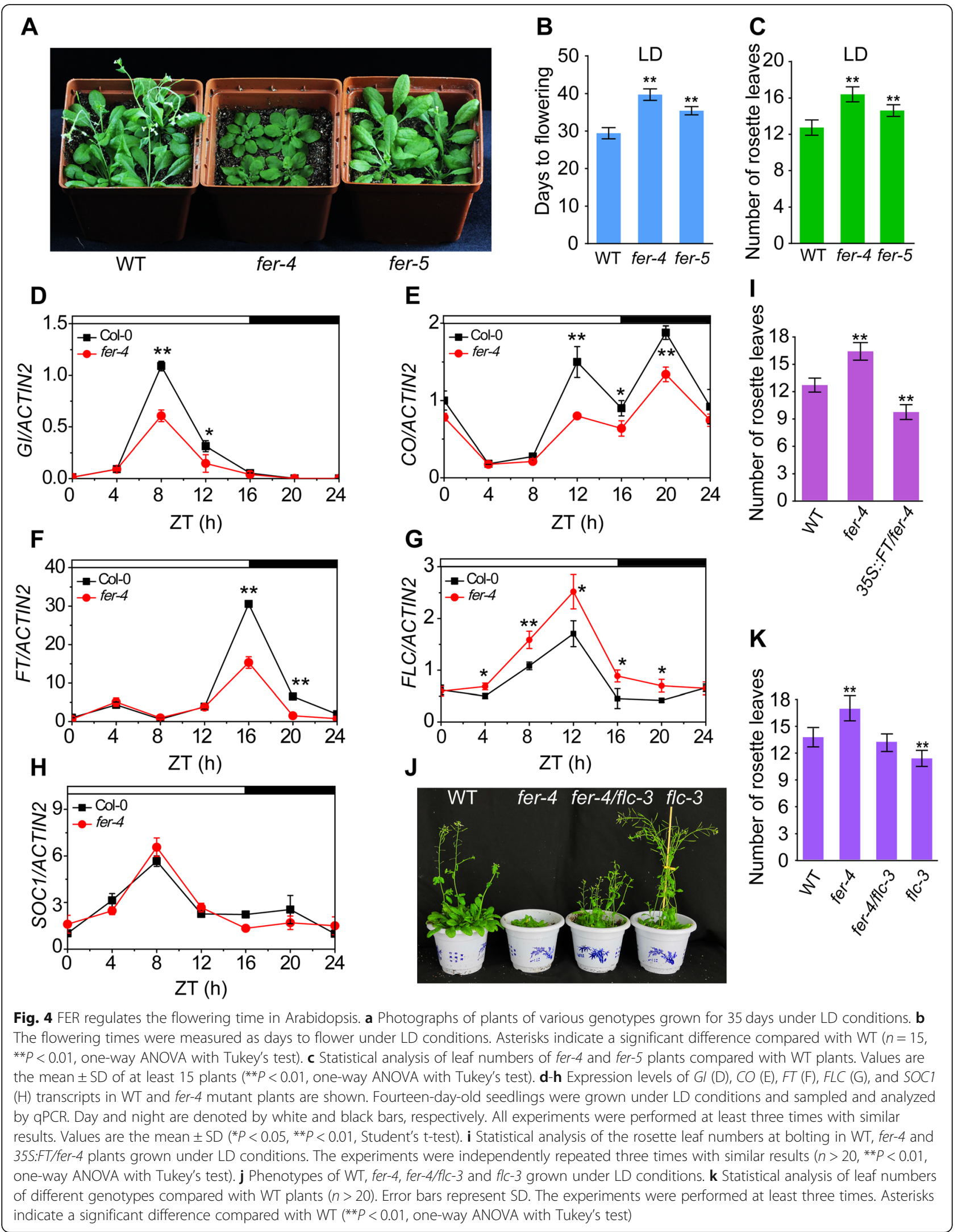


but the expression level of $\mathrm{CO}$ was lower in the mutant than in the WT (Fig. 4e), which led to the lower peak in $F T$ expression observed in the fer-4 mutant (Fig. 4f). To further confirm that FER regulates flowering mainly through the GI-CO-FT pathway, we examined the expression of two other key flowering factors, FLC and SOC1. FLC is a center node of the autonomous and vernalization pathways, and FLC expression was significantly elevated in the fer- 4 mutant compared with the WT plants, which indicated that either the autonomous or vernalization pathway is affected in the fer-4 mutant (Fig. 4g). In addition, the transcript level of SOC1 was unaffected in the fer-4 mutant (Fig. 4h). To confirm the genetic roles of FLC and FT that regulate flowering in the fer-4 mutant, we compared the flowering phenotypes among the Col-0, fer-4, 35S:FT/fer-4, flc-3 and fer-4/flc-3 plants. The flowering time of the 35S:FT/fer4 plant was earlier than those of the fer-4 mutant and WT plants (Fig. 4i), which indicated that FT expression could recover the late-flowering phenotype of the fer-4 mutant. Moreover, the loss of function of $F L C$ in the fer-4 mutant background alleviated the late-flowering phenotype of the fer-4 mutant (Fig. 4j-k), which indicated that FLC is involved in the FER-mediated acceleration of floral transition. Together, these results indicate that FER regulates flowering in Arabidopsis through multiple pathways.

\section{FER regulates the AS of some flowering-related genes}

We subsequently focused on the mechanism through which FLC expression is up-regulated in the fer- $4 \mathrm{mu}$ tant compared with the WT plants. There are two possible explanations for the higher expression of FLC observed in the fer- 4 mutant. First, the FER mutation might decrease the expression of some regulators of $F L C$ to release FLC expression, and second, the FER mutation might cause the abnormal splicing of FLC, resulting in an increase in functional FLC mRNA. To explore the silencing mechanism of $F L C$, we examined whether various FLC regulators, including FLOWERING CONTROL LOCUS A (FCA), FLOWERING LOCUS D (FLD), FY, VERNALIZATION 1 (VRN1) and $V R N 2$, were affected in the fer-4 mutant. The results showed that the mRNA expression levels of these genes showed only a slight little difference in the fer-4 mutant compared with the WT plants, which indicated that the increased FLC expression observed in the fer- 4 mutant was not caused by these genes (Fig. 5a). We also assessed the mRNA levels of three homologs of FLC, namely, MAF1, MAF2 and MAF3. The expression levels of these genes were significantly increased in the fer-4 mutant compared with the WT plants (Fig. 5b), which suggested that $M A F$ homologs likely act as targets of FER. We then measured the ratio of spliced to unspliced FLC transcripts to analyze the splicing efficiency of FLC introns 1 and 6 [44]. The splicing efficiency of both introns 1 and 6 was increased in the fer-4 mutant compared with the WT plants (Fig. $5 \mathrm{c}$ and Additional file 5: Figure S5). We predicted that the increased splicing efficiency of $F L C$ introns 1 and 6 would result in a decreased level of unspliced RNA but resulted in an increased level of spliced RNA. Thus, the increased level of spliced FLC RNA increases the expression of $F L C$ in the fer-4 mutant compared with the WT plants.

We performed an RNA-Seq analysis to identify additional potential target flower-related genes of FERmediated splicing. We generated more than 40 million reads, and $89.8 \%$ of the generated reads could be properly aligned to the TAIR10 reference genome (Additional file 6: Figure S6A). By plotting the coverage of reads along each transcript unit, we found a uniform distribution with no obvious $3^{\prime} / 5^{\prime}$ bias (Additional file 6: Figure S6B), and a comparison of the mapped reads to the gene model (TAIR10) revealed that approximately 99\% of the reads mapped to exons (Additional file 6: Figure $\mathrm{S6C}$ ), which indicated the high quality of the cDNA libraries. The RNA-Seq analysis revealed that 1753 and 1634 genes displayed higher (at least 2-fold with $P<$ 0.05 ) and lower transcript levels, respectively, in the fer4 compared with the Col-0 plants (Additional file 6: Figure S6D and Additional file 9: Table S1). Based on the RNA-Seq data, 2450 genes were identified as having splicing defects in the fer- 4 mutant compared with the WT control $(P<0.05$, inclusion level difference $>0.05$ or $<-$ 0.05 ), and these could be sorted into five categories: 1146 alternative 3' splice sites (A3'SS), 552 alternative 5' splice sites (A5'SS), 472 exon skipping (ES), 238 intron retained (IR) and 42 mutually exclusive exons (MXE) (Fig. 5d and Additional file 10: Table S2). A Gene Ontology term enrichment analysis revealed that different splicing genes are involved in RNA processing, response to salt stress and flower development regulation (Fig. 5e and Additional file 11: Table S3). We identified approximately 28 flower-related genes that might be involved in FER-mediated splicing, and these included MAF1, MAF2, FY and EMBRYONIC FLOWER 2 (EMF2) (Additional file 12: Table S4). To better visualize the differential splicing between fer-4 and WT in the floweringrelated pathway, we used heat maps of the genes showing splicing changes (Fig. 5f), which indicated that the splicing patterns were significantly altered in the fer- 4 mutant. Through semi-qPCR analysis, we also confirmed that some flowering-related genes (MAF1, MAF2, and $M A F 3$ ) exhibit splicing changes (Fig. $5 \mathrm{~g}$ and $\mathrm{h}$ and Additional file 13: Table S5). In summary, these results support a function of FER in regulating flowering in Arabidopsis by mediating AS. 


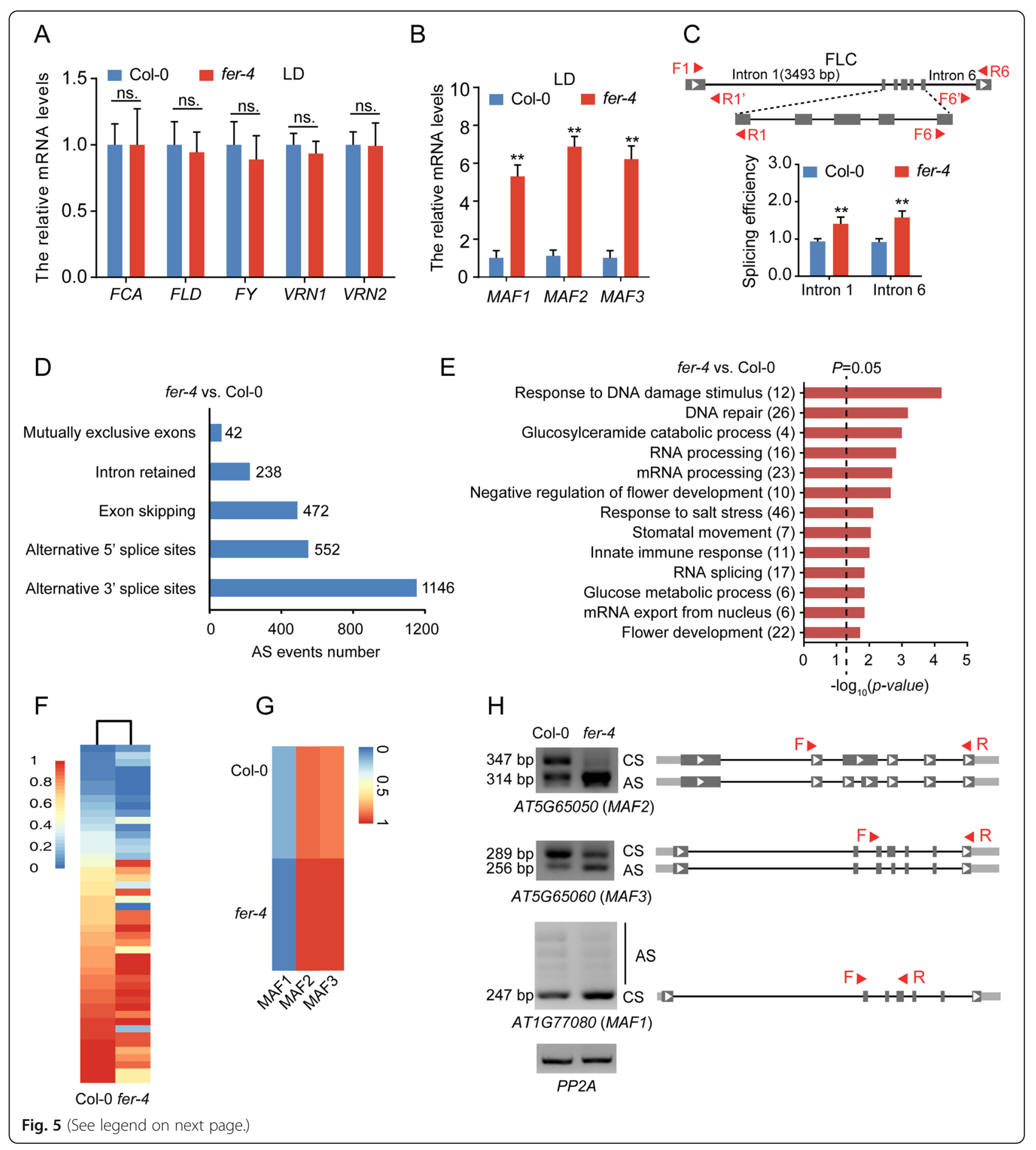


(See figure on previous page.)

Fig. 5 FER is required for pre-mRNA splicing of flowering-related genes in Arabidopsis. a-b Relative mRNA levels of WT and fer-4 mutant at ZT 4. LD, long day condition. ACTIN2 was used as an internal control gene. Error bars indicate the SD of three technical replicates. All experiments were performed at least three times (ns. means no significant; ${ }^{* *} P<0.01$, Student's t-test). c Genomic structure of FLC. The gray boxes represent the exons; black lines indicate introns; F, forward primer; R, reverse primer; The primer pairs F1/R1' and F6'/R6 were used to detect the unspliced RNA for FLC introns 1 and 6, respectively. F1/R1 and F6/R6 were used to detect spliced mRNA in wild-type and fer-4 mutants. ACTIN2 was used as an internal control. Seven-day-old Col-0 and fer-4 seedlings were grown under LD conditions and collected at ZT 12. Splicing efficiency (spliced/ unspliced) was calculated. The experiments were performed three times ( ${ }^{* *} P<0.01$, Student's t-test). $\mathbf{d}$ Numbers of differential splicing events between the fer-4 mutant and WT detected by RNA sequencing (RNA-Seq) analysis ( $n=3)$. e Gene Ontology (GO) enrichment of genes with significant splicing changes between fer- 4 and WT $(n=3)$. The black dotted line indicates $P=0.05$. The numbers indicated the representative genes involved in the pathway. $\mathbf{f}$ Heat maps of differential pre-mRNA splicing of flowering-related genes between fer-4 mutant and wild-type based on the exon inclusion levels detected by RNA-Seq analysis. $\mathbf{g}$ Heat maps of differential pre-mRNA splicing of flowering-related genes (MAF1, MAF2 and MAF3) based on the exon inclusion levels from RNA-seq data $(n=3)$. $\mathbf{h}$ Semi-quantitative PCR (semi-qPCR) validation of differential splicing events between fer-4 mutant and Col-0. Seven-day-old Col-0 and fer-4 seedlings were grown under LD conditions and collected at ZT 4. CS indicates constitutive splicing, and AS indicates alternative splicing. Red triangles show the position of primers used by semiqPCR (Supplemental Table 5). PP2A was used as the internal control. The experiments were independently repeated three times with similar results

\section{RALF1 peptide affects the flowering time under LD conditions}

FER functions as a receptor for RALF1 and RALF23 [31, 32], and in this study, FER was shown to be involved in the control of the AS of some flowering-related genes. We tested whether RALF1 or RALF23 might serve as a peptide hormone that alters the splicing pattern of flowering genes by activating FER. First, we analyzed the expression of RALF1 and RALF23 in ePlant and found that both were expressed in the shoot apex (Additional file 7: Figure S7A-B). A qPCR analysis further confirmed that RALF1 is expressed in the shoot (Additional file 7: Figure $\mathrm{S7C}$ ). We subsequently used RALF1 as an example to analyze the role of RALF peptides in the control of flowering. The WT and fer- 4 mutant plants were treated with the RALF1 peptide, and the splicing efficiencies of FLC introns 1 and 6 were detected. The efficiencies of the FLC introns 1 and 6 significantly increased after RALF1 treatment in Col-0 plants, whereas the alterations in the splicing efficiencies of FLC introns 1 and 6 caused by RALF1 treatment were significantly attenuated in the fer-4 mutant (Fig. 6a). To investigate whether the pre-mRNA splicing of MAFs is regulated by RALF1, we analyzed the splicing changes in MAFs with or without RALF1 treatment. The results showed that the splicing pattern of MAF1-3 was altered in the RALF1-treated plants, similar to the results obtained in the FER mutant plants, which indicated that FER and RALF1 might have opposite roles in regulating the AS of some flowering genes (Fig. 6b). We subsequently assessed the role of RALF1 in regulating the flowering time in Arabidopsis. First, we obtained two RALF1-overexpressing lines (RALF1-OX\#2 and RALF1$O X \# 3$ ) and identified two ralf1 mutants (ralf1-knockout and RALF1-RNAi-knockdown lines) (Additional file 8: Figure S8A-D). Under LD conditions, the two RALF1-OX lines showed a late-flowering phenotype compared with the WT line (Fig. 6c-d). In contrast, the ralf1 mutant and $R A L F 1-R N A i$ line displayed an early-flowering phenotype
(Fig. 6e-f). The mRNA levels of FLC increased in RALF1$O X$ and decreased in the ralf1 mutant, which was consistent with the flowering phenotype (Fig. $6 \mathrm{~g}$ ). In addition, the splicing efficiencies of introns 1 and 6 were increased in the RALF1-OX plants and decreased in the ralf1 mutant compared with WT. In summary, these results indicate that RALF1 also regulates the splicing of flowering genes and exerts an opposite effect on the flowering time compared with FER.

\section{Discussion}

Several environmental signals entrain the circadian oscillator. In this study, we found that FER acts not only as a simple output of the clock but also as a regulator of clock genes. FER mutation affects the period length and amplitude of circadian outputs (Fig. 3a-f and Additional file 3: Figure S3A-D) and thus represses the downstream GI transcript and further delays the flowering time partly through the CO-FT pathway (Fig. 7). FER is also involved in other flowering pathways, primarily by repressing FLC and FLC-related MAFs (Fig. 7).

The circadian clock coordinates responses to multiple environmental challenges that cannot be avoided by a sessile plant. More importantly, the strength of many cell signaling pathways is regulated by the circadian clock, and this process is known as circadian gating [45]. Thus, the circadian clock is important for plant growth and fitness. Here, we showed that FER fluctuates along with the diurnal rhythm (Fig. 1a-b). Why does FER exhibit a rhythm in plant cells? FER is a versatile regulator of plant growth and stress responses $[28,37]$ and acts as a critical node for the crosstalk of extracellular or intracellular cues [29]. We propose that the diurnal rhythm of FER might function as an important output of circadian gating and confers a fitness advantage. For example, FER mediates the inhibition of the immune response in plants [32], and its mRNA nadir occurs at approximately midnight and dawn, which are times that are mainly 


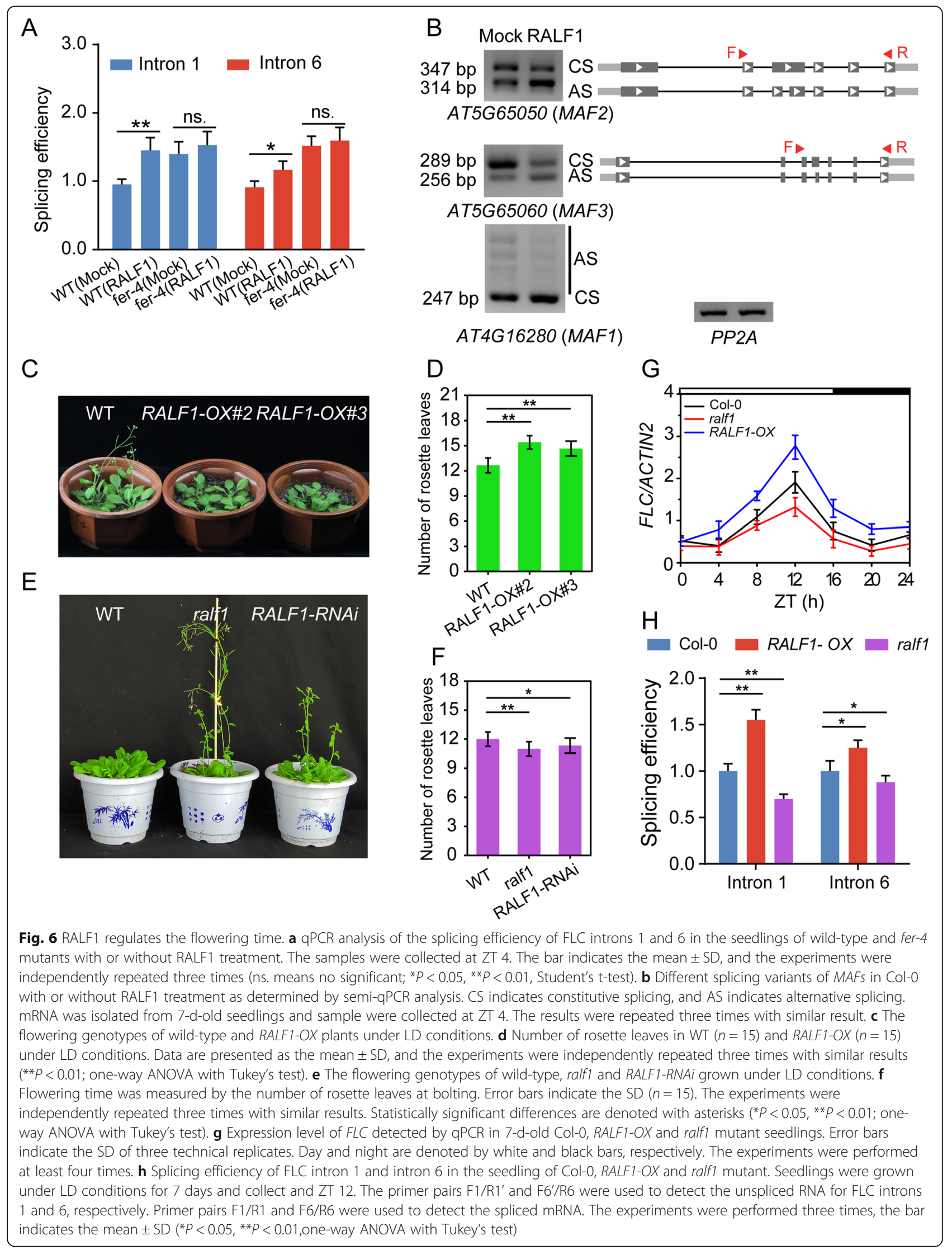




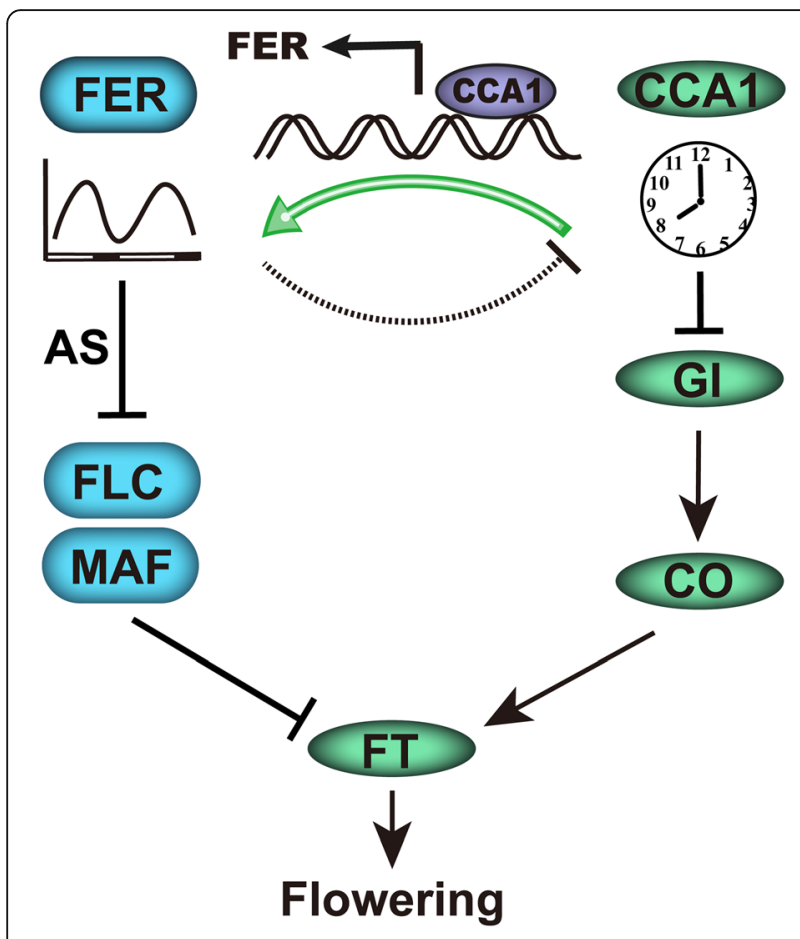

Fig. 7 Proposed working model of FER function in Arabidopsis flowering. FER fluctuated at a diurnal rhythm along with FER, and the clock oscillator CCA1 appears to bind the FER chromatin and promote its expression. In turn, FER will down-regulate CCA1 expression and regulate flowering through the Gl-CO pathway. Thus, FER-CCA1 appears to form an interlocking feedback loop during flowering time control. In addition, FER is a negative regulator of FLC and MAF by regulating their splicing and transcript accumulation. Thus, FER functions in flowering transition by splicing pre-mRNA of genes and regulating expression of flowering-related genes. Arrows denote activation, and bars indicate repression. Details are provided in the text

characterized by sporulation and spore dissemination $[46,47]$. This finding is consistent with the hypothesis that the FER diurnal rhythm might be involved in the defense response. In addition, starch accumulates in daytime and degrades at night, and FER also regulates starch metabolism in leaves [48]. We observed that the expression of FER begins to increase at dawn, peaks at ZT 4, and then decreases at dusk (Fig. 1a), which is consistent with the hypothesis that the diurnal rhythm of FER might also be related to starch metabolism. More importantly, the diurnal rhythm of FER might endow FER with the ability to balance the vegetative and reproductive growth times in response to changes in environmental signaling.

Some studies have indicated that RALFs play opposite roles in certain tissues and/or environmental responses compared with FER. For example, RALF22/23-overexpressing transgenic plants and fer- 4 mutants display similar retarded growth phenotypes and increased sensitivity to salt stress [37]. RALF1 and FER play negative and positive roles in leaf growth, respectively [33, 49]. Here, we found that RALF1 and FER have different roles in flowering and the AS of certain mRNAs. One possible reason for this phenomenon is that FER might recruit distinct downstream factors to fulfill its context-specific roles. In addition, RALF23, as another ligand of FER [32], is also expressed in the shoot apex. Further studies should explore whether RALF23 and FER play the same roles in flowering regulation.

The AS of pre-mRNA is an important regulatory mechanism. A few proteins or signaling pathways reportedly regulate AS in plants. Our study suggests that the RALF1 peptide influences the AS of floweringrelated genes through FER and further regulates the flowering time. However, the mechanisms used by the RALF1-FER pathway to regulate mRNA AS remain poorly understood. In addition, the mechanism through which the AS of many mRNAs is regulated by the RALF1-FER axis and the physiological significance of RALF1-FER-regulated mRNA AS remain unknown, and these processes should be examined in further studies.

\section{Conclusions}

Here, we found that FER, which is up-regulated by CCA1, not only outputs the clock but also regulates clock genes. This protein controls the vegetative-reproductive growth transition, likely by regulating the mRNA expression and AS of flowering-related genes, including FLC and its homologs (MAFs).

\section{Methods}

\section{Plant growth conditions}

Arabidopsis (Columbia ecotype) was used as the WT in all experiments unless stated otherwise. The fer-4, flc-3 mutant, 35S::FT, Ubi::FER-Flag (FER-OX) and srn have been previously described [27, 43, 50]. CCA1-OX was provided by Paul P. Dijkwe [51], and the ralf1 mutant (SALK_036331) [52] was obtained from ABRC and confirmed using specific primers (Additional file 8: Figure S8A-B and Additional file 13: Table S5). For the Ubi:: RALF1-Flag transgenic plants (RALF1-OX), the fulllength CDS of RALF1 was sub-cloned into the pCAMBIA 1301 vector under the control of the $U b i$ promoter through homologous recombination of the attB1 and attB2 sites (Gateway recombination cloning technology). The RALF1-OX lines were generated using the Agrobacterium-mediated floral dip method and identified using specific primers (Additional file 8: Figure S8C-D and Additional file 13: Table S5).

For analysis of clock gene expression, the seeds were planted on 1/2 MS medium supplemented with $1.0 \%$ sucrose and $0.8 \%$ agar ( $\mathrm{pH} \mathrm{5.8)}$ at $4{ }^{\circ} \mathrm{C}$ for 3 days in the dark. The seeds were then transferred to either LD (16-h light/8-h-dark) or SD (8-h-light/16-h-dark) conditions at 
a light intensity of 50 to $60 \mu \mathrm{mol} \mathrm{m}{ }^{-2} \mathrm{~S}^{-1}$ and a constant temperature of $22^{\circ} \mathrm{C}$. Seven-day-old seedlings grown under $\mathrm{LD} / \mathrm{SD}$ condition and then transferred to constant light conditions at ZT 24 for FER rhythm analysis.

\section{RNA extraction and qPCR analyses}

For the mRNA expression analyses, the samples were harvested and powdered in liquid nitrogen. Total RNA was extracted using the TRIzol reagent (Ambion, 15, 596-026) and digested using DNase I (TaKaRa) to remove the genomic DNA. cDNA was synthesized from $1000 \mathrm{ng}$ of total RNA by using a cDNA synthesis kit (Fermentas, K1622). qPCR was performed using Mx3000P (Stratagene) with SYBR Premix Ex Taq II (TaKaRa). The primers used for the qPCR analysis are listed in Additional file 13: Table S5, and ACTIN2 was used as an internal reference in the circadian experiments. The cDNAs were amplified following denaturation using 42 -cycle programs $\left(95^{\circ} \mathrm{C}, 15 \mathrm{~s} ; 60{ }^{\circ} \mathrm{C}, 20 \mathrm{~s}\right.$ per cycle).

The expression of the FER-related gene subfamily was investigated using the web-based tool "Diurnal", the gene expression data under SD were according to COL_SD (Arabidopsis Col-0 grown under circadian conditions of $\mathrm{SD}$ ), and the gene expression data under LD were according to long day conditions (Arabidopsis Ler strain, grown under the circadian conditions of LD). The gene expression data under LL were according to LL23 LDHH [Arabidopsis Col-0 grown under the circadian conditions light $(12 \mathrm{~h})$, light $(12 \mathrm{~h}) / \mathrm{hot}(24 \mathrm{~h})$, and subjected to light $(24 \mathrm{~h})]$. The period and amplitude of the clock genes were analyzed using BioDare2 (https://biodare2.ed.ac.uk) and the FFT NNLS method [41].

\section{Semi-qPCR analyses}

Seven-day-old Col-0 and fer- 4 seedlings were grown at $22^{\circ} \mathrm{C}$ under LD conditions and collected at ZT 4. For the RALF1 peptide treatment experiment, 7-d-old seedlings were treated with $1 / 2$ liquid MS in the presence or absence of $1 \mu \mathrm{M}$ RALF1 for $3 \mathrm{~h}$, then collected at ZT 4 and powdered in liquid nitrogen for RNA extraction. For the semiquantitative PCR (semi-qPCR) analysis of the AS of the flowering genes (e.g., MAF1, MAF2, and $M A F 3)$, Protein phosphatase $2 A(P P 2 A)$ was used as a reference gene as described in the RNA splicing experiments [53]. The primers for FLC intron retention 1 and 6 were according to Xiong et al. [22]. Semi-qPCR mixes were prepared using $2 \times$ Master Mix (TsingKe, TSE004) according to the manufacturer's instructions. Reactions were performed with initial incubation at $95^{\circ} \mathrm{C}$ for 10 min followed by 26 cycles of $15 \mathrm{~s}$ at $95^{\circ} \mathrm{C}, 30 \mathrm{~s}$ at $55^{\circ} \mathrm{C}$ and $1 \mathrm{~min} / \mathrm{kb}$ at $72{ }^{\circ} \mathrm{C}$. The semi-qPCR products were electrophoresed on $1.5 \%$ agarose gels and stained with ethidium bromide. The primer sequences used for the
semi-qPCR analysis are shown in Additional file 13: Table S5.

\section{Determination of the flowering time}

For the rosette leaf measurement, the plants were grown in a randomized fashion on soil under LD conditions at $22^{\circ} \mathrm{C}$ in a greenhouse with light intensity conditions of $80-100 \mu \mathrm{mol} \mathrm{m}^{-2} \mathrm{~S}^{-1}$. More than 40 plants of each genotype were planted for each independent experiment. The flowering time was determined according to the visible flower buds at the center of the rosette and the days from germination to flowering from three biological replicates.

\section{Western blot analysis}

For analysis of the protein levels under LD conditions, the WT and $p F E R:: F E R$-GFP plants were planted on $1 / 2$ MS medium for $7 \mathrm{~d}$. The samples were harvested and powdered in liquid nitrogen. Next, $200 \mathrm{mg}$ of the seed-

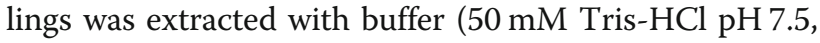
$150 \mathrm{mM} \mathrm{NaCl}, 5.0 \%$ glycerin, EDTA-2Na, 1.0\% Triton $\mathrm{X}-100)$ for $30 \mathrm{~min}$ and then separated by $10.0 \%$ SDSPAGE to detect the two forms of FER (phosphorylated and non-phosphorylated) in one band. In contrast to our previous study, we did not add glycerol to the SDSPAGE gel [27], and we shortened the running time to easily survey the total FER level in a single band. The gel was blotted onto an NC filter membrane using tank transfer. The blots were blocked with 5.0\% defatted milk for $1 \mathrm{~h}$ at $4{ }^{\circ} \mathrm{C}$ with agitation, incubated with anti-GFP antibody (CMC, 1:5000) and FER antibody (1:3000) for $4-5 \mathrm{~h}$ at RT with agitation, and then washed 3 times for 7 min each with TBS-T at RT with agitation. The blots were incubated with a secondary antibody diluted 1 : 10000 in milk for $1 \mathrm{~h}$ at RT with agitation for enhanced chemiluminescence detection (Thermo Scientific, 34, 075). $\beta$-actin was used as the loading control.

\section{Gene cloning and dual-LUC analysis}

Genomic DNA was extracted from Arabidopsis leaves using the DNeasy Plant Mini Kit (Qiagen, 69,104), the promoter of FER ( $p F E R$ ) was cloned from the genomic DNA, and the coding region of the CCA1 gene was cloned from the cDNA. The gene-specific primers used for the PCR analysis are listed in Additional file 13: Table S5. The amplification product of $p F E R$ was cloned into pGreenII-LUC at the BamHI and NcoI sites to add the firefly LUC reporter gene, and CCA1 was cloned into pEGAD to generate the 35S::CCA1-pEGAD effector [54]. In addition, the pGreenII-LUC vector carrying Renilla (REN) LUC under the control of the $35 \mathrm{~S}$ promoter served as an internal control. The binding ability of CCA1 to the FER promoter was assessed according to the ratio of LUC to REN. The reporter and effector 
vectors were co-transformed into Arabidopsis protoplasts using the PEG method as previously described [43]. After incubation at $22^{\circ} \mathrm{C}$ for $24 \mathrm{~h}$, the transformed protoplasts were assayed for LUC and REN using dual LUC assay kits (Promega, E1910). The readouts of LUC and REN were assessed using Fluoroskan Ascent FL (Thermo Scientific, China) according to the manufacturer's instructions.

\section{EMSA}

For the EMSA, recombinant proteins of GST-CCA1 were expressed in Escherichia coli BL21 and subsequently purified using GST beads. The DNA-protein binding reaction was performed by incubating $20 \mathrm{fmol}$ of FITC-labelled probe with $5 \mu \mathrm{g}$ purified GST-CCA1 protein. For the competition experiments, $5 \mu \mathrm{g}$ purified GST-CCA1 protein and $100 \times$ unlabeled competitor A or $100 \times$ nonspecific competitor $\mathrm{B}$ were incubated in binding buffer $(100 \mathrm{mM}$ Tris- $\mathrm{HCl} \mathrm{pH}=8.0,5 \mathrm{mM}$ DTT, 2.5 mM EDTA, $0.25 \%$ Triton X-100 and $25 \%$ glycerin) for $15 \mathrm{~min}$, followed by the addition of $20 \mathrm{fmol}$ of FITClabeled probe for $20 \mathrm{~min}$. Next, the binding reaction mixture was loaded onto the 4\% PAGE gel (without SDS), which was resolved in $0.5 \times \mathrm{TBE}$ buffer for $40 \mathrm{~min}$ and then exposed to a fluorescence imager plate.

\section{ChIP assays}

The ChIP assay was performed as previously described [55]. Briefly, 3-week-old Col-0 and 35S::CCA1-Myc grown on soil under LD conditions were collected at ZT 0 and treated with $37 \mathrm{~mL}$ extraction buffer $1(0.4 \mathrm{M}$ sucrose; $10 \mathrm{mM}$ Tris- $\mathrm{HCl}, \mathrm{pH} 8.0 ; 5 \mathrm{mM} \quad \beta-\mathrm{ME} ; 0.1 \mathrm{mM}$ PMSF) containing $1 \%$ formaldehyde under a vacuum for $15 \mathrm{~min}$. A final concentration of $0.125 \mathrm{M}$ glycine was added to quench the crosslinking, and the vacuum was applied for an additional $5 \mathrm{~min}$. The plants were rinsed twice with water, ground to a powder with liquid nitrogen, and homogenized in extraction buffer $1[0.4 \mathrm{M}$ sucrose; $10 \mathrm{mM}$ Tris- $\mathrm{HCl}, \mathrm{pH} 8.0 ; 5 \mathrm{mM} \quad \beta$-ME; $0.1 \mathrm{mM}$ PMSF; complete protease inhibitor cocktail tablets (Roche)]. The filtered solution was centrifuged at $2100 \mathrm{~g}$ for $20 \mathrm{~min}$ at $4{ }^{\circ} \mathrm{C}$, and the pellet was resuspended in 1.5 $\mathrm{mL}$ of extraction buffer 2 [0.25 M sucrose; $10 \mathrm{mM}$ Tris$\mathrm{HCl} \mathrm{pH} \mathrm{8.0;} 10 \mathrm{mM} \mathrm{MgCl}_{2}, 1 \%$ Triton X-100; $0.1 \mathrm{mM}$ PMSF; $5 \mathrm{mM} \beta$-ME; complete protease inhibitor cocktail tablets (Roche)]. The samples were centrifuged at 12000 $\mathrm{g}$ for $10 \mathrm{~min}$ at $4{ }^{\circ} \mathrm{C}$ and lysed in Nuclei Lysis Buffer [50 mM Tris- $\mathrm{HCl} \mathrm{pH}$ 8.0, $10 \mathrm{mM}$ EDTA, 1\% SDS, $0.1 \mathrm{mM}$ PMSF, and protease inhibitor cocktail tablets (Roche)]. The chromatin solution was sonicated to shear the DNA to $200-600 \mathrm{bp}$, the sonicated chromatin suspension was centrifuged at $12000 \mathrm{~g}$ for $5 \mathrm{~min}$ and the supernatant was diluted 10-fold in ChIP Dilution Buffer [16.7 mM Tris- $\mathrm{HCl} \mathrm{pH}$ 8.0, $167 \mathrm{mM} \mathrm{NaCl}, 1.1 \%$ Triton X-100, 1.2
mM EDTA, $0.1 \mathrm{mM}$ PMSF, complete protease inhibitor cocktail tablets (Roche)]. The anti-Myc antibody was pre-bound to protein $\mathrm{A} / \mathrm{G}$ magnetic beads, mixed with the chromatin solution and incubated overnight at $4{ }^{\circ} \mathrm{C}$. The beads were then washed with Low Salt Buffer (50 $\mathrm{mM}$ Tris- $\mathrm{HCl} \mathrm{pH} 8.0,150 \mathrm{mM} \mathrm{NaCl}, 0.2 \%$ SDS, 0.5\% Triton X-100, 2 mM EDTA), High Salt Buffer (20 mM Tris- $\mathrm{HCl} \mathrm{pH} 8.0,500 \mathrm{mM} \mathrm{NaCl}, 0.2 \%$ SDS, $0.5 \%$ Triton $\mathrm{X}-100,2 \mathrm{mM}$ EDTA), LiCl Washing Buffer $(20 \mathrm{mM}$ Tris- $\mathrm{HCl} \mathrm{pH} 8.0,0.25 \mathrm{M} \mathrm{LiCl}, 1 \% \mathrm{NP}$ 40, $1 \%$ sodium deoxycholate, $1 \mathrm{mM}$ EDTA) and TE Washing Buffer (10 $\mathrm{mM}$ Tris- $\mathrm{HCl} \mathrm{pH} 8.0,1 \mathrm{mM}$ EDTA). The chromatin fragments were eluted with Elution Buffer $(50 \mathrm{mM}$ Tris$\mathrm{HCl} \mathrm{pH}$ 8.0, $10 \mathrm{mM}$ EDTA, 1\% SDS) and incubated at $65^{\circ} \mathrm{C}$ for $12 \mathrm{~h}$. The eluate was treated with proteinase $\mathrm{K}$ to digest proteins. The DNA was purified with the DNA purification kit, and $50 \mu \mathrm{l}$ TE Buffer was added to elution. $1 \mu \mathrm{l}$ DNA sample was used for qPCR, and the primers used for $\mathrm{qPCR}$ are listed in Additional file 13: Table S5.

\section{Splicing efficiency of FLC measurement}

The splicing efficiency of FLC was determined as previously [44]. Briefly, $5 \mu \mathrm{g}$ of total RNA was reversetranscribed into cDNA, the cDNA was used as template in qPCR to amplify FLC intron 1 spliced with primer F1 and R1, which cover the splicing junction; FLC intron 6 spliced with primer F6 and R6, which cover the splicing junction; FLC intron 1 unspliced with primer F1 and R1', FLC intron 6 unspliced with primer F6' and R6. Splicing efficiency was calculated by the level of spliced RNA normalized to the level of unspliced RNA.

\section{RNA-seq analyses}

Seven-day-old Col-0 and fer-4 seedlings grown under LD conditions and collected at ZT 4 were used for total RNA extraction. Total RNA was extracted with the mirVana miRNA Isolation Kit (Ambion, AM1561) following the manufacturer's protocol. RNA integrity was evaluated using the Agilent 2100 Bioanalyzer (Agilent Technologies, Santa Clara, CA, USA). The samples with an RNA Integrity Number (RIN) $\geq 7$ were subjected to the subsequent analysis. The libraries were constructed using the TruSeq Stranded mRNA LT Sample Prep Kit (Illumina, San Diego, CA, USA) according to the manufacturer's instructions. Illumina sequencing was performed in Shanghai OE Biotech. Co., Ltd. with the Illumina sequencing platform (Illumina HiSeq X Ten).

Raw data (raw reads) were processed by using the NGS QC Toolkit [56]. The low quality reads were removed. The remaining reads were mapped to the Arabidopsis TAIR10 genome by using hisat2 [57]. Splicing events were identified by rMATS, and classification was obtained according to Matlin et al. $[58,59] . P$ value < 
0.05 and inclusion level difference $>0.05$ or $<-0.05$ were set as the threshold for significant differential splicing changes. The FPKM value of each gene was calculated using cufflinks [60], and the read counts of each gene were obtained by htseq-count [61]. Differentially expressed genes (DEGs) were identified using the DESeq (2012) functions estimateSizeFactors and nbinomTest. $P$ value $<0.05$ and fold change $>2$ or fold change $<0.5$ were set as the threshold for significantly differential expression. Hierarchical cluster analysis of DEGs was performed to explore gene expression patterns. The raw RNA-seq data were uploaded to the NCBI database with the accession number SRX5988587.

\section{Statistics}

Any significant differences in data were analyzed by Student's t-test or by multivariate comparison (one-way ANOVA) using SPSS (version 17.0) software. All statistical tests were clearly described in the figure legends and/or in the methods section.

\section{Supplementary information}

Supplementary information accompanies this paper at https://doi.org/10. 1186/s12870-019-2223-y.

Additional file 1: Figure S1. Expression levels of FER under LD, SD and $\mathrm{LL}$. conditions using the web-based tool Diurnal Expression of FER was associated with the Arabidopsis Columbia strain 0 grown under SD circadian conditions. Expression of FER under LD conditions was associated with Arabidopsis Ler grown under LD circadian conditions. Expression of FER under LL conditions was associated with Arabidopsis Col-0 grown under the circadian conditions of light (12 h, $12 \mathrm{~h}$, and $24 \mathrm{~h})$.

Additional file 2: Figure S2. Chromatin of several CrRLK1L subfamily genes containing the EE motif (A) The CCA1-bound EE motifs. (B) Sequences containing the EE motif in the chromatin of several CrRLKIL subfamily genes. The number indicates the length of the sequence starting upstream of the ATG start codon.

Additional file 3: Figure S3. fer-4 mutant alters the amplitudes of certain clock genes (E) qPCR analysis of CCA1 (A), LHY (B), TOC1 (C) and PRR7 (D) expression levels in WT and fer-4 mutant plants under LD. The 7-day-old seedlings were harvested at 3-h intervals. Day and night are denoted by white and black bars, respectively. The amplitude was analyzed using BioDare2. All experiments were performed at least three times with similar results, and the error bars indicate the SD of three technical replicates $\left({ }^{*} P<0.05,{ }^{* *} P<0.01\right.$, Student's t-test).

Additional file 4: Figure S4. Loss of FER delays flowering in Arabidopsis (A) The flowering genotypes of the WT (C24) and srn mutant (another FER null mutant) under LD conditions. The plants were grown in soil under light conditions at an intensity of $50 \mu \mathrm{mol} \mathrm{m}-2 \mathrm{~S}-1$ for $45 \mathrm{~d}$. A representative experiment of three independent replicates is shown. (B) The flowering times measured as days to flower under LD conditions. Values are the mean \pm SD of at least 15 plants. The asterisk indicates a significant difference $\left({ }^{* *} P<0.01\right.$, one-way ANOVA with Tukey's test). (C) Number of rosette leaves in WT $(n=15)$ and $\operatorname{srn}(n=15)$ under $\mathrm{LD}$ conditions. The bar indicates the SD ( ${ }^{*} P<0.01$, one-way ANOVA with Tukey's test).

Additional file 5: Figure S5. The relative mRNA levels of unspliced FLC intron 1 and intron 6 decreased in fer-4 mutant. Total RNA were extracted from 7-d-old seedling grown under LD condition and collected at ZT 12. The primer pairs F1/R1' and F6'/R6 were used to detect the unspliced RNA for $F L C$ introns 1 and 6, respectively. Primer pairs F1/R1 and F6/R6 were used to detect the spliced mRNA. The experiments were performed three times the bar indicates the mean \pm SD $\left({ }^{*} P<0.01\right.$, Student's t-test).
Additional file 6: Figure S6. Quality analyses of RNA-seq data from wild-type and fer-4 mutant (A) Mapping results of RNA reads. (B) Distribution of RNA-seq read coverage in the Col-0 and fer-4 mutant were plotted along the length of the transcriptional unit. The $X$-axis indicates the percentile of the gene body, and the $y$-axis shows the read number. (C) Distribution of the RNA-seq reads along annotated Arabidopsis genomic features in Col-0 and fer-4. Among the mapped reads, more than $99 \%$ of reads map to the annotated exon. (D) Summary of genes whose transcripts were upregulated or downregulated in the fer-4 mutant as determined by RNA-seq experiments.

Additional file 7: Figure S7. QPCR analysis of RALF1 mRNA levels in different tissues RALF1 (A), RALF23 (B) expression patterns as illustrated from the bar website: http://bar.utoronto.ca/. (C) qPCR analysis of RALF mRNA showed that RALF1 is highly expressed in root, and also expressed in shoot apex. RNA were extracted from 10-day-old root, shoot apex and leaves. The expression of RALF1 in leaves is lower compare to other two tissue types. ACTIN2 was used as an internal control.

Additional file 8: Figure S8. Isolation and characterization of the ralf 1 mutant and RALF1-OX lines (A) Verification of the location of the T-DNA insertion described in SIGnAL (http://signal.salk.edu/cgi-bin/tdnaexpress). For the ATG start codon, the black boxes are exons, and the white boxes are the UTR. The exact sites of the T-DNA insertions (indicated by triangles) were mapped by PCR and DNA sequencing of the PCR products. (B) The T-DNA insert was present in the ralf1 mutant but not in the WT genomic DNA. (C) The relative mRNA levels of the RALF1 genes in the WT and eight different RALF1-OX lines. ACTIN2 was used as the internal control to calculate the relative mRNA levels. The experiments were performed at least three times with similar results. (D) Transgenic RALF1-OX lines were verified by PCR. WT plants were used as a negative control.

Additional file 9: Table S1. Genes with significant ( $>$ 2-fold, $p<0.05$ ) expression in fer-4 compare with WT as determined by RNA-seq anaylsis.

Additional file 10: Table S2. Genes with alternative splicing events in fer-4 plant as determined by RNA-seq analysis.

Additional file 11: Table S3. Enrichment analysis showing the enriched categories forFER knockout compared with WT.

Additional file 12: Table S4. Flowering relate genes with alternative splicing events in fer-4 plant as determined by RNA-seq analysis.

Additional file 13: Table S5. Primers list.

\section{Abbreviations}

AS: Alternative Splicing; CCA1: CIRCADIAN CLOCK-ASSOCIATED1; CCA1OX: CCA1-overexpressing; CO: CONSTANS; DEGs: Differentially expressed genes; EC: evening complex; ELF3: EARLY FLOWERING 3; EMF2: EMBRYONIC FLOWER 2; FCA: FLOWERING CONTROL LOCUS A; FER: FERONIA;

FLC: FLOWERING LOCUS C; FLD: FLOWERING LOCUS D; FLM: FLOWERING LOCUS M; FT: FLOWERING LOCUS T; GI: GIGANTEA; HERK1: HERCULES1; LD: Long day; LHY: LATE ELONGATED HYPOCOTYL; LL: Continuous light; LUX: LUX ARRHYTHMO; MAF: MADS AFFECTING FLOWERING; PP2A: Protein phosphatase 2A; PRMT5: Protein arginine methyltransferase 5; PRR7: PSEUDORESPONSE REGULATOR7; PRR9: PSEUDO-RESPONSE REGULATOR9;

RALF: Rapid Alkalinization Factor; SC35: The 35-kDa splicing factor; SD: Short day; snRNPs: Small nuclear ribonucleoprotein particles; SOC1: SUPPRESSOR OF CONSTANS 1; SR: Serine/arginine-rich; SVP: SHORT VEGETATIVE PHASE; THE1: THESEUS 1; TOC1: TIMING OF CAB EXPRESSION 1;

VRN1: VERNALIZATION 1; ZT: Zeitgeber time

\section{Acknowledgements}

We thank Hongtao Liu, Paul P. Dijkwe, and Alice Cheung for providing the mutants used in this study.

\section{Source of seeds}

The fer-4, flc-3 mutant, 355::FT, Ubi::FER-Flag (FER-OX) and srn have been previously described [27, 43, 50]. CCA1-OX was provided by Paul P. Dijkwe [51], and the ralf1 mutant (SALK_036331) was obtained from ABRC.

\section{Authors' contributions}

FY and LW conceived the project and designed research; LW, TY, BW and XL performed research; QL, and FY contributed new reagents/analytic tools; FY, 
LW and SL analyzed data and wrote the paper; all authors reviewed and approved the manuscript for publication.

\section{Funding}

This work was supported by grants from the National Natural Science Foundation of China (NSFC-31571444, 31400232).

\section{Availability of data and materials}

All data generated or analysed during this study are included in this published article and its supplementary information files.

\section{Ethics approval and consent to participate}

Not applicable.

\section{Consent for publication}

Not applicable.

\section{Competing interests}

The authors declare that they have no competing interests.

\section{Author details}

${ }^{1}$ College of Biology, State Key Laboratory of Chemo/Biosensing and Chemometrics, and Hunan Province Key Laboratory of Plant Functional Genomics and Developmental Regulation, Hunan University, Changsha 410082, People's Republic of China. 'National Engineering Laboratory for Rice and By-product Deep Processing, Central South University of Forestry and Technology, Changsha 410004, People's Republic of China. ${ }^{3}$ National Key Laboratory of Plant Molecular Genetics, Chinese Academy of Sciences Center for Excellence in Molecular Plant Sciences, Institute of Plant Physiology and Ecology, Shanghai Institutes for Biological Sciences, Chinese Academy of Sciences, Shanghai 200032, People's Republic of China. ${ }^{4}$ Department of Plant and Microbial Biology, University of California, Berkeley, CA 94720, USA.

\section{Received: 7 October 2019 Accepted: 30 December 2019}

\section{Published online: 16 January 2020}

\section{References}

1. Greenham K, McClung CR. Integrating circadian dynamics with physiological processes in plants. Nat Rev Genet. 2015;16(10):598-610.

2. Cui Z, Xu Q, Wang X. Regulation of the circadian clock through pre-mRNA splicing in Arabidopsis. J Exp Bot. 2014;65(8):1973-80.

3. Harmer SL. The circadian system in higher plants. Annu Rev Plant Biol. 2009; 60:357-77.

4. Nakamichi N, Kiba T, Henriques R, Mizuno T, Chua NH, Sakakibara H. PSEUDO-RESPONSE REGULATORS 9, 7, and 5 are transcriptional repressors in the Arabidopsis circadian clock. Plant Cell. 2010;22(3):594-605.

5. Farre EM, Harmer SL, Harmon FG, Yanovsky MJ, Kay SA. Overlapping and distinct roles of PRR7 and PRR9 in the Arabidopsis circadian clock. Curr Biol. 2005;15(1):47-54.

6. Alabadi D, Oyama T, Yanovsky MJ, Harmon FG, Mas P, Kay SA. Reciprocal regulation between $\mathrm{TOC} 1$ and $\mathrm{LHY} / \mathrm{CCA} 1$ within the Arabidopsis circadian clock. Science. 2001;293(5531):880-3.

7. Gendron JM, Pruneda-Paz JL, Doherty CJ, Gross AM, Kang SE, Kay SA. Arabidopsis circadian clock protein, TOC1, is a DNA-binding transcription factor. Proc Natl Acad Sci U S A. 2012;109(8):3167-72.

8. Huang W, Perez-Garcia P, Pokhilko A, Millar AJ, Antoshechkin I, Riechmann $J$, Mas P. Mapping the core of the Arabidopsis circadian clock defines the network structure of the oscillator. Science. 2012;336(6077):75-9.

9. Nusinow DA, Helfer A, Hamilton EE, King JJ, Imaizumi T, Schultz TF, Farre EM, Kay SA. The ELF4-ELF3-LUX complex links the circadian clock to diurnal control of hypocotyl growth. Nature. 2011;475(7356):398-402.

10. Herrero E, Kolmos E, Bujdoso N, Yuan Y, Wang M, Berns MC, Uhlworm H, Coupland G, Saini R, Jaskolski M, et al. EARLY FLOWERING4 recruitment of EARLY FLOWERING3 in the nucleus sustains the Arabidopsis circadian clock. Plant Cell. 2012;24(2):428-43.

11. Fowler S, Lee K, Onouchi H, Samach A, Richardson K, Morris B, Coupland G, Putterill J. GIGANTEA: a circadian clock-controlled gene that regulates photoperiodic flowering in Arabidopsis and encodes a protein with several possible membrane-spanning domains. EMBO J. 1999;18(17):4679-88.
12. Suarez-Lopez P, Wheatley K, Robson F, Onouchi H, Valverde F, Coupland G. CONSTANS mediates between the circadian clock and the control of flowering in Arabidopsis. Nature. 2001;410(6832):1116-20.

13. Bohlenius H, Huang T, Charbonnel-Campaa L, Brunner AM, Jansson S, Strauss SH, Nilsson O. CO/FT regulatory module controls timing of flowering and seasonal growth cessation in trees. Science. 2006;312(5776):1040-3.

14. Song YH, Shim JS, Kinmonth-Schultz HA, Imaizumi T. Photoperiodic flowering: time measurement mechanisms in leaves. Annu Rev Plant Biol. 2015;66:441-64.

15. Valverde F, Mouradov A, Soppe W, Ravenscroft D, Samach A, Coupland G. Photoreceptor regulation of CONSTANS protein in photoperiodic flowering. Science. 2004;303(5660):1003-6.

16. Onouchi H, Igeno MI, Perilleux C, Graves K, Coupland G. Mutagenesis of plants overexpressing CONSTANS demonstrates novel interactions among Arabidopsis flowering-time genes. Plant Cell. 2000;12(6):885-900.

17. Samach A, Onouchi H, Gold SE, Ditta GS, Schwarz-Sommer Z, Yanofsky MF, Coupland G. Distinct roles of CONSTANS target genes in reproductive development of Arabidopsis. Science. 2000;288(5471):1613-6.

18. Michaels SD, Amasino RM. FLOWERING LOCUS C encodes a novel MADS domain protein that acts as a repressor of flowering. Plant Cell. 1999;11(5): 949-56.

19. Crevillen $P$, Dean C. Regulation of the floral repressor gene FLC: the complexity of transcription in a chromatin context. Curr Opin Plant Biol. 2011;14(1):38-44.

20. Yan Q, Xia X, Sun Z, Fang Y. Depletion of Arabidopsis SC35 and SC35-like serine/arginine-rich proteins affects the transcription and splicing of a subset of genes. PLoS Genet. 2017;13(3):e1006663.

21. Wu Z, Zhu D, Lin X, Miao J, Gu L, Deng X, Yang Q, Sun K, Zhu D, Cao XF. RNA-binding proteins at RZ-1B and at RZ-1C play a critical role in regulation of pre-mRNA splicing and gene expression during Arabidopsis development. Plant Cell. 2015:28(1):55-73.

22. Xiong F, Ren JJ, Yu Q, Wang YY, Lu CC, Kong LJ, Otegui MS, Wang XL. AtU2AF65b functions in abscisic acid mediated flowering via regulating the precursor messenger RNA splicing of ABI5 and FLC in Arabidopsis. New Phytol. 2019. https://doi.org/10.1111/nph.15756.

23. Moore MJ, Proudfoot NJ. Pre-mRNA processing reaches back to transcription and ahead to translation. Cell. 2009:136(4):688-700.

24. Rosloski SM, Singh A, Jali SS, Balasubramanian S, Weigel D, Grbic V. Functional analysis of splice variant expression of MADS AFFECTING FLOWERING 2 of Arabidopsis thaliana. Plant Mol Biol. 2013;81(1-2):57-69.

25. Posé $D$, Verhage $L$, Ott $F$, Yant L, Mathieu J, Angenent GC, Immink RGH, Schmid M. Temperaturedependent regulation of flowering by antagonistic FLM variants. Nature. 2013;503(7476):414-7.

26. Zhang Z, Zhang S, Zhang Y, Wang X, Li D, Li Q, Yue M, Li Q, Zhang YE, XU $Y$, Xue $Y$, Chong K, Bao S. Arabidopsis floral initiator SKB1 confers high salt tolerance by regulating transcription and pre-mRNA splicing through altering histone H4R3 and small nuclear ribonucleoprotein LSM4 methylation. Plant Cell. 2011;23(1):396-411.

27. Chen J, Yu F, Liu Y, Du C, Li X, Zhu S, Wang X, Lan W, Rodriguez PL, Liu X, et al. FERONIA interacts with ABI2-type phosphatases to facilitate signaling cross-talk between abscisic acid and RALF peptide in Arabidopsis. Proc Natl Acad Sci U S A. 2016:113(37):E5519-27.

28. Nissen KS, Willats W, Malinovsky FG. Understanding CrRLK1L function: cell walls and growth control. Trends Plant Sci. 2016:21(6):516-27.

29. Liao H, Tang R, Zhang X, Luan S, Yu F. FERONIA receptor kinase at the crossroads of hormone signaling and stress responses. Plant Cell Physiol. 2017:58(7):1143-50.

30. Ge Z, Dresselhaus T, Qu L. How CrRLK1L receptor complexes perceive RALF signals. Trends Plant Sci. 2019;24(11):978-81.

31. Haruta M, Sabat G, Stecker K, Minkoff BB, Sussman MR. A peptide hormone and its receptor protein kinase regulate plant cell expansion. Science. 2014; 343(6169):408-11

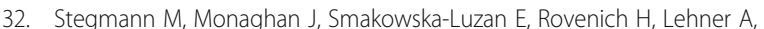
Holton N, Belkhadir Y, Zipfel C. The receptor kinase FER is a RALF-regulated scaffold controlling plant immune signaling. Science. 2017:355(6322):287-9.

33. Cheung AY, Wu HM. THESEUS 1, FERONIA and relatives: a family of cell wallsensing receptor kinase? Curr Opin Plant Biol. 2011;14(6):632-41.

34. Duan Q, Kita D, Li C, Cheung AY, Wu HM. FERONIA receptor-like kinase regulates $\mathrm{RHO}$ GTPase signaling of root hair development. Proc Natl Acad Sci U S A. 2010;107(41):17821-6.

35. Feng W, Kita D, Peaucelle A, Cartwright HN, Doan V, Duan Q, Liu MC, Maman J, Steinhorst L, Schmitz-Thom I, et al. The FERONIA receptor kinase 
maintains Cell-Wall integrity during salt stress through $\mathrm{Ca}(2+)$ signaling. Curr Biol. 2018;28(5):666-75.

36. Guo H, Nolan TM, Song G, Liu S, Xie Z, Chen J, Schnable PS, Walley JW, Yin Y. FERONIA receptor kinase contributes to plant immunity by suppressing Jasmonic acid signaling in Arabidopsis thaliana. Curr Biol. 2018;28(20):331624.

37. Zhao C, Zayed O, Yu Z, Jiang W, Zhu P, Hsu CC, Zhang L, Tao WA, LozanoDuran $\mathrm{R}$, Zhu JK. Leucine-rich repeat extensin proteins regulate plant salt tolerance in Arabidopsis. Proc Natl Acad Sci U S A. 2018;115(51):13123-8.

38. Mockler TC, Michael TP, Priest HD, Shen R, Sullivan CM, Givan SA, McEntee C, Kay SA, Chory J. The DIURNAL project: DIURNAL and circadian expression profiling, model-based pattern matching, and promoter analysis. Cold Spring Harb Symp Quant Biol. 2007;72:353-63.

39. Nagel DH, Doherty CJ, Pruneda-Paz JL, Schmitz RJ, Ecker JR, Kay SA. Genome-wide identification of CCA1 targets uncovers an expanded clock network in Arabidopsis. Proc Natl Acad Sci U S A. 2015;112(34):E4802-10.

40. Kamioka M, Takao S, Suzuki T, Taki K, Higashiyama T, Kinoshita T, Nakamichi N. Direct repression of evening genes by CIRCADIAN CLOCK-ASSOCIATED1 in the Arabidopsis circadian clock. Plant Cell. 2016;28(3):696-711.

41. Zielinski T, Moore AM, Troup E, Halliday KJ, Millar AJ. Strengths and limitations of period estimation methods for circadian data. PLoS One. 2014 9(5):e96462.

42. Lu SX, Webb CJ, Knowles SM, Kim SH, Wang Z, Tobin EM. CCA1 and ELF3 interact in the control of hypocotyl length and flowering time in Arabidopsis. Plant Physiol. 2012;158(2):1079-88.

43. Yu F, Qian L, Nibau C, Duan Q, Kita D, Levasseur K, Li X, Lu C, Li H, Hou C, et al. FERONIA receptor kinase pathway suppresses abscisic acid signaling in Arabidopsis by activating ABI2 phosphatase. Proc Natl Acad Sci U S A. 2012; 109(36):14693-8.

44. Marquardt S, Raitskin O, Wu Z, Liu F, Sun Q, Dean C. Functional consequences of splicing of the antisense transcript COOLAIR on FLC transcription. Mol Cell. 2014;54(1):156-65.

45. Seo PJ, Mas P. STRESSing the role of the plant circadian clock. Trends Plant Sci. 2015;20(4):230-7

46. Slusarenko AJ, Schlaich NL. Downy mildew of Arabidopsis thaliana caused by Hyaloperonospora parasitica (formerly Peronospora parasitica). Mol Plant Pathol. 2003;4(3):159-70.

47. Wang W, Barnaby JY, Tada Y, Li H, Tor M, Caldelari D, Lee DU, Fu XD, Dong $X$. Timing of plant immune responses by a central circadian regulator. Nature. 2011:470(7332):110-4.

48. Yang T, Wang L, Li C, Liu Y, Zhu S, Qi Y, Liu X, Lin Q, Luan S, Yu F. Receptor protein kinase FERONIA controls leaf starch accumulation by interacting with glyceraldehyde-3-phosphate dehydrogenase. Biochem Biophys Res Commun. 2015;465(1):77-82

49. Bergonci T, Ribeiro B, Ceciliato PH, Guerrero-Abad JC, Silva-Filho MC, Moura DS. Arabidopsis thaliana RALF1 opposes brassinosteroid effects on root cell elongation and lateral root formation. J Exp Bot. 2014;65(8):2219-30.

50. He R, Li X, Zhong M, Yan J, Ji R, Li X, Wang Q, Wu D, Sun M, Tang D, et al. A photo-responsive F-box protein FOF2 regulates floral initiation by promoting FLC expression in Arabidopsis. Plant J. 2017;91(5):788-801.

51. Lai AG, Doherty CJ, Mueller-Roeber B, Kay SA, Schippers JH, Dijkwel PP. CIRCADIAN CLOCK-ASSOCIATED 1 regulates ROS homeostasis and oxidative stress responses. Proc Natl Acad Sci U S A. 2012;109(42):17129-34.

52. Du C, Li X, Chen J, Chen W, Li B, Li C, Wang L, Li J, Zhao X, Lin J, Liu X, Luan S, Yu F. Receptor kinase complex transmits RALF peptide signal to inhibit root growth in Arabidopsis. Proc Natl Acad Sci U S A. 2016;113(51):E8326-34.

53. James AB, Syed NH, Bordage $S$, Marshall J, Nimmo GA, Jenkins Gl, Herzyk P, Brown JW, Nimmo HG. Alternative splicing mediates responses of the Arabidopsis circadian clock to temperature changes. Plant Cell. 2012;24(3): 961-81.

54. Hellens RP, Allan AC, Friel EN, Bolitho K, Grafton K, Templeton MD, Karunairetnam S, Gleave AP, Laing WA. Transient expression vectors for functional genomics, quantification of promoter activity and RNA silencing in plants. Plant Methods. 2005;1:13.

55. Liu H, Yu X, Li K, Klejnot J, Yang H, Lisiero D, Lin C. Photoexcited CRY2 interacts with $\mathrm{ClB} 1$ to regulate transcription and floral initiation in Arabidopsis. Science. 2008;322(5907):1535-9.

56. Patel RK, Jain M. NGS QC toolkit: a toolkit for quality control of next generation sequencing data. PLoS One. 2012;7(2):e30619.

57. Kim D, Langmead B, Salzberg SL. HISAT: a fast spliced aligner with low memory requirements. Nat Methods. 2015;12(4):357-60.
58. Shen S, Park JW, Lu ZX, Lin L, Henry MD, Wu YN, Zhou Q, Xing Y. rMATS: robust and flexible detection of differential alternative splicing from replicate RNA-Seq data. Proc Natl Acad Sci U S A. 2014;111(51):E5593-601.

59. Matlin AJ, Clark F, Smith CW. Understanding alternative splicing: towards a cellular code. Nat Rev Mol Cell Biol. 2005;6(5):386-98.

60. Trapnell C, Roberts A, Goff L, Pertea G, Kim D, Kelley DR, Pimentel H, Salzberg SL, Rinn JL, Pachter L. Differential gene and transcript expression analysis of RNA-seq experiments with TopHat and cufflinks. Nat Protoc. 2012;7(3):562-78.

61. Anders S, Pyl PT, Huber W. HTSeq--a Python framework to work with highthroughput sequencing data. Bioinformatics. 2015;31(2):166-9.

\section{Publisher's Note}

Springer Nature remains neutral with regard to jurisdictional claims in published maps and institutional affiliations.
Ready to submit your research? Choose BMC and benefit from:

- fast, convenient online submission

- thorough peer review by experienced researchers in your field

- rapid publication on acceptance

- support for research data, including large and complex data types

- gold Open Access which fosters wider collaboration and increased citations

- maximum visibility for your research: over $100 \mathrm{M}$ website views per year

At BMC, research is always in progress.

Learn more biomedcentral.com/submissions 